\title{
Surgical shortcuts with commentary
}

\section{Training in a simple, low-cost, inanimate box model improves the laparoscopic skills}

\author{
Clevin L, Grantcharov TP. \\ Does box model training improve surgical dexterity \\ and economy of movement during virtual reality \\ laparoscopy? A randomised trial. Acta Obstet Gynecol \\ Scand. 2008; 87: 99-103.
}

Objective: Laparoscopic box-model trainers have been used in training curricula for a long time; however, data on their impact on skills acquisition are still limited. Our aim was to validate a low-cost box model trainer as a tool for the training of skills relevant to laparoscopic surgery. Methods: Randomised, controlled trial (Canadian Task Force Classification I). Results: Sixteen gynaecologic residents with limited laparoscopic experience were randomised to a group that received a structured box-model training curriculum and a control group. Performance before and after the training was assessed in a virtual reality laparoscopic trainer (LapSim) and was based on objective parameters, registered by the computer system (time, error, and economy of motion scores). Group A showed significantly greater improvement in all performance parameters compared with the control group: economy of movement $(p=0.001)$, time $(p=0.001)$ and tissue damage ( $p=0.036$ ), confirming the positive impact of box-trainer curriculum on laparoscopic skills acquisition. Conclusions: Structured laparoscopic skill training on a lowcost box-model trainer improves performance as assessed using the VR system. Trainees who used the box-model trainer showed significant improvement compared to the control group. Box-model trainers are valid tools for laparoscopic skills training and should be implemented in the comprehensive training curricula in gynaecology.

\section{COMMENTARY}

The authors conducted a randomised control trial (RCT) to evaluate the efficacy of an inanimate box-trainer model for laparoscopic skills acquisition.
The study was performed on 16 residents in OB\&GYN with limited experience in laparoscopy. Participants were randomised to a group that received structured training in the box model for $3 \mathrm{~h}$ or to a group that did not receive any training (control group).

Both groups performed at the beginning of the study and 7 days later standardised training tasks (camera navigation, instrument navigation, coordination, grasping, lifting and grasping, cutting, and clip applying) on a validated virtual reality laparoscopic trainer model (LapSim). These tasks were objectively evaluated by the computer for the following parameters: time, error and economy of motion.

The group that underwent the training in the box model showed significantly greater improvement in all parameters evaluated in the LapSim compared with the control group. The data are consistent with previous reports and confirm that training in a simple, low-cost, inanimate box model improves the laparoscopic skills.

The article is, however, somewhat limited since the raw data are not displayed. Indeed, the authors do not show the baseline data of both groups in the LapSim, nor the data at the end of the study, making difficult to draw conclusions about the inter-group differences at the beginning and at the end of the study and the intra-group differences (the effect of training). Furthermore, the "structured" training is not described in detail besides the total duration (i.e. $3 \mathrm{~h}$ ).

Nevertheless, with all these limitations the study clearly indicates the importance of training; moreover, that this can be done in a box model. Such a model has the disadvantage, as discussed by the authors, that usually requires the direct and permanent observation of the tutor, which is time-consuming and expensive. This factor, however, can be modified by applying alternative scoring methods. On the other hand, the box model has the major advantage of being more realistic in terms of laparoscopic instruments and tactile feedback, and significantly cheaper than the computerised models, making them more suitable for universal implementation. 
Although many publications have sufficiently demonstrated that both virtual reality and box models are efficient for training laparoscopic skills, and that they have face, content and construct validity, their concurrent and predictive validity remains largely unknown and demands further studies.

\section{Roger Molinas, Asuncion}

\section{Difficult to set up strict guidelines after one case report}

\section{Buzaglo K, Bruchim I, Lau SK, Ferenczy A, Tulandi T, Gotlieb WH. \\ Sarcoma post-embolization for presumed uterine fibroids. Gynecol Oncol. 2008; 108: 244-7.}

Background: Uterine artery embolization has increasingly been used in the last decade as a conservative treatment approach for uterine fibroids. Rarely, sarcomas have been diagnosed shortly after uterine artery embolization. It remains unclear whether a change in diagnostic work-up is required prior to uterine artery embolization in order to avoid missing sarcomas and delaying definitive treatment. Case: A 45-yearold underwent uterine artery embolization for symptomatic uterine fibroids. Six months later, following progressive symptoms, she underwent surgery, which revealed an endometrial stromal sarcoma. This manuscript raises the issue and reviews the existing literature concerning the need of tissue diagnosis prior to uterine artery embolization. Conclusion: Assessing the risk of malignancy by taking into account the clinical symptoms, physical exam, and imaging findings is essential prior to uterine artery embolization.

\section{COMMENTARY}

The incidence of uterine sarcomas is $1 / 1,000$ fibroids and in order to diagnose this condition, we need a true cut biopsy from the fibroid and even so we could not be sure to have the sarcoma. A problem with a true cut biopsy would also be contamination during the procedure; as a consequence we should take out the uterus in toto if sarcoma is suspected. Magnetic resonance imaging (MRI) and ultrasound are not reliable diagnostic tools; however, suspicion can be made, and in these cases, adequate diagnostic workout should be utilized, although most leiomyosarcomas arise independently. Rapid growth is not a reliable indication (a retrospective review of 580 sarcomas $<3 \%$ had a rapidly enlarged uterus and one leiomyosarcoma was found in 371 women operated for rapidly growing leiomyomas [1]). More than 100,000 uterine artery embolizations have been performed worldwide.
Therefore, strict guidelines is difficult to set up after one case report.

1. Parker WH, Fu YS, Berek JS (1994) Uterine sarcoma in patients operated on for presumed leiomyoma and rapidly growing leiomyoma. Obstet Gynecol 83:414-418

Olav Istre, Oslo

\section{The choice to use a specific tool should be made according to the specific procedure to be performed}

Berg A, Sandvik L, Langebrekke A, Istre O. Fertil Steril. A randomized trial comparing monopolar electrodes using glycine $1.5 \%$ with two different types of bipolar electrodes (TCRis, Versapoint) using saline, in hysteroscopic surgery. 2008 Mar 25 [Epub ahead of print].

Objective: To compare three types of equipment during hysteroscopic resection. Methods: In a randomized study, 200 premenopausal women with menorrhagia caused by dysfunctional bleedings, fibroids, or polyps hysteroscopic resection was performed either with monopolar electrodes using glycine $1.5 \%$ as irrigant or with two different types of bipolar electrodes (TCRis; Olympus, Hamburg, Germany and Versapoint; Gynecare, Menlo Park, CA) using saline as irrigant. The change in serum sodium as a result of irrigant consumption, operating time, and amount of tissue removed were estimated. Results: A statistically significant reduction in mean serum sodium from $138.7 \mathrm{mmol} / 1$ to $133.8 \mathrm{mmol} /$ 1 was seen in the monopolar group, compared with the case of the saline groups with no reduction. The amount of resected tissue in the monopolar and TCRis group was approximately $1.00 \mathrm{~g} / \mathrm{min}$, compared with $0.65 \mathrm{~g} / \mathrm{min}$ in the Versapoint group. Loss of fluid during the procedure was significantly higher in the two bipolar groups. Conclusions: Bipolar electrodes appear to have a safer profile compared with monopolar electrodes because of the unchanged serum sodium. Irrigant consumption was significantly higher in the two bipolar groups, without any side effects during or after the procedure. Furthermore, the TCRis loop appears to be superior to the Versapoint loop, as regards operating time and amount of tissue removed.

\section{COMMENTARY}

The study was aimed to compare in a randomized fashion 200 pre-menopausal women with menorrhagia three types of equipment during hysteroscopic resection, i.e., monopolar electrodes using glycine $1.5 \%$ as irrigant or with two different types of bipolar electrodes using saline as irrigant. According 
to serum sodium change and irrigant consumption, the authors concluded that bipolar electrodes appear to have a safer profile and that TCRis loop is superior to the Versapoint loop in terms of operating time and amount of tissue removed.

I recommend reading this very interesting study since it is one of the few randomized controlled studies available in literature on this issue. Unfortunately, in order to conclude if a procedure is really more effective than another, the clinical trial should base its conclusion on a clinically significant reduction of complications and, thus, of patients' morbidity. On the other hand, the serum sodium change, the irrigant consumption, the operating time, and the amount of tissue removed are only surrogate end-point of safety and efficacy. I think that, in expert hands, both procedures are related with a very low rate of complications and that the choice to use a specific tool should be made according to the specific procedure to be performed. In particular, Colacurci et al. [2] demonstrated that bipolar electrodes is associated with shorter operative time and lower complication rate than unipolar electrodes for treating septate uterus, whereas Muzii et al. [3] concluded the higher efficacy of unipolar over bipolar electrodes for endometrial polyps greater than $2 \mathrm{~cm}$.

The article is classified as: important confirmation.

\section{References}

1. Berg A, Sandvik L, Langebrekke A, Istre O (2008) A randomized trial comparing monopolar electrodes using glycine $1.5 \%$ with two different types of bipolar electrodes (TCRis, Versapoint) using saline, in hysteroscopic surgery Fertil Steril Mar 25 [Epub ahead of print]

2. Colacurci N, De Franciscis P, Mollo A, Litta A, Perino A, Cobellis L, De Placido G (2007) Small-diameter hysteroscopy with Versapoint versus resectoscopy with unipolar knife for the treatment of septate uterus: a prospective randomized study. J Minim Invasive Gynecol 14:622-627

3. Muzii L, Bellati F, Pernice M, Manci N, Angioli R, Panici PB (2007) Resectoscopic versus bipolar electrode excision of endometrial polyps: a randomized study. Fertil Steril 87:909-917

\section{Fulvio Zullo, Naples}

\section{Bipolar thermal ablation was superior to balloon ablation after a 5-year follow up, but should we stop using thermal balloon?}

Kleijn JH, Engels R, Bourdrez P, Mol BW, Bongers MY. Five-year follow-up of a randomised controlled trial comparing NovaSure and ThermaChoice endometrial ablation. BJOG 2008; 115: 193-198.
Objective: We have previously reported that NovaSure was more effective than balloon ablation at 12 months followup in the treatment of menorrhagia. In this paper, we report the 5-year outcome of this study. The objective was to evaluate amenorrhoea rates, hysterectomy rate, and quality of life associated with the bipolar impedance-controlled endometrial ablation technique (NovaSure) in comparison with balloon ablation technique (ThermaChoice) at 5 years after administration. Methods: NovaSure and ThermaChoice were compared in a total of 126 premenopausal women suffering from menorrhagia without intracavitary abnormalities. Women were randomly allocated to bipolar radio-frequency ablation and balloon ablation in a 2:1 ratio. The main outcome measures were amenorrhoea rate, hysterectomies, and health-related quality of life (HRQol) as reported at 5-year follow-up. Results: At 5 years of follow-up, the total response rate was $96 \%$ in the bipolar group and $90 \%$ in the balloon group. Amenorrhoea was reported in the bipolar group by $48 \%$ of women and in the balloon arm by $32 \%$ (relative risk 1.6 [.93-2.6]). There were eight women in the bipolar group (9.8\%) and five in the balloon group (12.9\%) who had undergone a hysterectomy. Furthermore, there was a significant equal improvement of HRQoL over time in both groups. Conclusions: At 5 years of follow-up, bipolar thermal ablation was superior over balloon ablation in the treatment of menorrhagia.

\section{COMMENTARY}

Second generation endometrial ablation has firmly established itself as an important option in the management of menorrhagia. A number of different devices and energy modalities are available to help the surgeon destroy the endometrium. Most of the clinical trials in second generation relate to single or multi-centre experience of a single device; such trials are often performed for regulatory approval. There is a real shortage of comparative trials and indeed in the UK an important regulatory body-NICE - has called for further studies comparing difference devices.

Against this background it is very good to see this important paper from Bonger's unit in the Netherlands who describe a randomised trial between two important market leaders in endometrial ablation, Novasure and ThermaChoice. The study is well powered, a double-blind RCT and has good outcome measures including amenorrhoea, hysterectomy and increasingly important health-related quality-of-life indicators. An important strength of this study is the relatively long-term (by endometrial ablation trial standards) follow-up of 5 years. This is extremely important as it is only by longterm follow-up can we really assess the true results of endometrial ablation in the clinical setting.

The results suggest that Novasure-bipolar thermal ablation-was superior to balloon ablation after a 5-year 
follow-up as evidenced by a higher amenorrhoea rate and a lower hysterectomy rate in the Novasure arm. Interestingly, however, the quality-of-life improvement was similar in both groups.

Should this study on its own prompt us to stop using thermal balloon? The answer is no. There are many factors determining the success after endometrial ablation not least of all cavity size, endometrial thickness, presence of fibroids and patient age. This study, however, does give some very interesting pointers and findings and it should be remembered that the ThermaChoice device has had some modification since this trial was performed, which may make more meaningful comparison with the current ThermaChoice product difficult.

Ellis Downes, Enfield 